W. Kohnen and G. Mason

Nagoya Math. J.

Vol. 192 (2008), 119-136

\title{
ON GENERALIZED MODULAR FORMS AND THEIR APPLICATIONS
}

\author{
WINFRIED KOHNEN AND GEOFFREY MASON*
}

\begin{abstract}
We study the Fourier coefficients of generalized modular forms $f(\tau)$ of integral weight $k$ on subgroups $\Gamma$ of finite index in the modular group. We establish two Theorems asserting that $f(\tau)$ is constant if $k=0, f(\tau)$ has empty divisor, and the Fourier coefficients have certain rationality properties. (The result is false if the rationality assumptions are dropped.) These results are applied to the case that $f(\tau)$ has a cuspidal divisor, $k$ is arbitrary, and $\Gamma=\Gamma_{0}(N)$, where we show that $f(\tau)$ is modular, indeed an eta-quotient, under natural rationality assumptions on the Fourier coefficients. We also explain how these results apply to the theory of orbifold vertex operator algebras.
\end{abstract}

\section{$\S 1$. Introduction}

Let $\Gamma \subseteq S L(2, \mathbf{Z})$ be a subgroup of finite index. A generalized modular function of weight zero (GMF) on $\Gamma$ is a function $f(\tau)$ holomorphic throughout the complex upper half-plane $\mathcal{H}$, satisfying

$$
f(\gamma \tau)=\chi(\gamma) f(\tau), \quad \gamma \in \Gamma,
$$

for some character $\chi: \Gamma \rightarrow \mathbf{C}^{*}$, and which is 'meromorphic at the cusps'. As usual, the latter condition means that for all $\gamma \in S L(2, \mathbf{Z})$, there is a $q$-expansion

$$
f(\gamma \tau)=\sum_{n=n_{0}}^{\infty} a_{n} q^{n / M}
$$

for some positive integer $M$ (depending on $\gamma$ ), convergent in a punctured neighborhood of the origin in the $q$-plane. (Here and below, $q=e^{2 \pi i \tau}$ for $\tau \in \mathcal{H}$.) Thus $f$ enjoys all of the properties of a (classical) modular function of weight zero on $\Gamma$, except that $\chi$ is not required to be unitary. That is, we allow the possibility that $|\chi(\gamma)| \neq 1$ for $\gamma \in \Gamma$, and even if $\chi$ is unitary it

Received April 3, 2007.

Revised November 14, 2007.

2000 Mathematics Subject Classification: 11F03, 11F99, 17B69.

*Supported by DMS0245225 and the Committee on Research at UC Santa Cruz. 
may not have finite order. There is an obvious extension of (1) to the case of integral weight $k$ that we will also use.

The general study of GMFs was initiated in [KM1], where a GMF in the above sense was called a PGMF ( $\mathrm{P}$ for parabolic). Throughout the present paper, we use the definition given above. Several ways to construct GMFs are described in [KM1]. For large enough weight $k$ and for $\chi$ parabolic (loc. cit.), the Eisenstein series

$$
\sum_{\gamma} \chi(\gamma)^{-1}(c \tau+d)^{-k}
$$

is a GMF of weight $k$ (as usual, $\gamma$ ranges over a certain set of elements of $\Gamma$ with second row $(c, d)$ ). One may then multiply it by a classical form of weight $-k$ to obtain a GMF of weight zero. Also, the logarithmic derivative of a GMF of weight zero is a (classical) meromorphic modular form $g(\tau)$ of weight 2, and conversely one can construct GMFs of weight zero by exponentiating an antiderivative of a suitable weight 2 classical form. This second construction is considered in more detail below.

Aside from their intrinsic interest, one of the main motivations for studying GMFs is the possibility of applying them to so-called 'Moonshine' properties of finite groups in orbifold theory. The Conway-Norton conjecture $[\mathrm{CN}]$, which concerned the association of certain modular functions of weight zero to elements of the Monster simple group $M$, was established by Borcherds $[\mathrm{B}]$ in a celebrated paper. In particular, the $q$-expansions of $[\mathrm{CN}]$ were shown to be both hauptmoduln on genus zero subgroups of $S L(2, \mathbf{R})$, and also graded traces of elements of $M$ acting on the Frenkel-LepowskyMeurman Moonshine Module $V^{\natural}$ [FLM]. A fundamental fact underlying this construction (loc. cit.) is that $V^{\natural}$ is a particular type of vertex operator algebra.

Since the work of Borcherds, it has become clear that one should expect similar results to hold in great generality. Precisely, if $V$ is a rational vertex operator algebra and $g$ an automorphism of $V$ of finite order, one expects that an appropriately defined notion of graded trace of $g$ on $V$ will be a modular function of weight zero on some congruence subgroup of $S L(2, \mathbf{Z})$. (We refer the reader to [DM1] and Section 4 below for more background. A detailed knowledge of vertex operator algebras and orbifolds will not be needed in order to understand the results of the present paper.) From this perspective, the case $V=V^{\natural}$ is distinguished in that the graded traces are 
hauptmoduln, a fact which will certainly not hold in general. Borcherds' proof cannot, and does not, generalize, so if one wants to understand the more general situation, a new approach is needed. If successful, such an approach would - when applied to the Moonshine Module - give a new proof of the fact that the trace functions of elements in $M$ over $V^{\natural}$ are modular functions without necessarily showing that they are in fact hauptmoduln. There are several paths down which one might travel in pursuit of this goal. The one we advocate here, based on GMFs, is particularly arithmetic.

A general theory of graded traces of automorphisms of vertex operator algebras was developed in [DLM]. One of the results obtained there (although not stated as such) is that if $V$ is $a^{1}$ holomorphic vertex operator algebra (an example is $V^{\natural}$ ) and $g$ an automorphism of $V$ of finite order, then the graded trace of $g$ on $V$ is a GMF on a congruence subgroup. This leads to the following conjecture:

Suppose that $f$ is a nonzero GMF on $\Gamma_{0}(N)$ with $q$-expansion

$f(\tau)=\sum a_{n} q^{n}$ with leading nonzero coefficient equal to 1 and

Fourier coefficients $a_{n} \in \mathbf{Z}$. Then $f$ is a classical modular function.

One may - and in Section 2 we shall - contemplate stronger conjectures of a similar type. The general idea is that a GMF which is not a classical modular function has 'poorly behaved' Fourier coefficients. In any case, we will see that (2) already implies the modularity of trace functions of elements of $M$ acting on $V^{\natural}$. In the present paper we make a start on Conjecture (2) and certain variations.

As we already mentioned, it was observed in [KM1] that if $f$ is a GMF of weight zero on $\Gamma$ then the logarithmic derivative $f^{\prime} / f$ is a (classical) meromorphic modular form of weight 2 on $\Gamma$, and conversely any meromorphic modular form of weight 2 satisfying some mild conditions arises in this way. In particular, holomorphic modular forms of weight 2 on $\Gamma$ correspond in this way to GMFs which have all of their poles and zeros located at the cusps of $\Gamma$, and cusp-forms of weight 2 correspond to GMFs with no poles and no zeros (in $\mathcal{H} \cup \mathbf{Q} \cup \infty$ ). Thus, if $\Gamma$ has positive genus then it supports nonconstant GMFs with empty divisor. It is this circumstance which

\footnotetext{
${ }^{1}$ It is standard terminology to call a VOA holomorphic if it is simple and has a unique irreducible module. This is quite different from more familiar uses of the word holomorphic, for example in reference to a modular form.
} 
animates the main results of the present paper. A basic tool in the theory of modular functions is the fact that a modular function with no poles or zeros is constant, and as we have explained this generally fails for GMFs. One needs a substitute, and our first two main results each provide one, under somewhat different assumptions. Theorem 1 says that a GMF with no poles or zeros is constant if, in addition, it satisfies the integrality conditions of (2). In effect, Conjecture (2) is true if $f$ has no zeros or poles, moreover the proof applies to arbitrary subgroups of $\Gamma \subseteq S L(2, \mathbf{Z})$ of finite index in place of $\Gamma_{0}(N)$. Theorem 2 shows that the same result also holds under the weaker assumption that the Fourier coefficients are rational and are $p$-integral for almost all primes $p$, as long as $\Gamma$ is a congruence subgroup.

With these results in hand, we take up Conjecture (2) in case the poles and zeros of $f$ are at the cusps of $\Gamma_{0}(N)$. More precisely, we show (Theorem 3) that a GMF $f$ with Fourier coefficients satisfying the same integrality conditions as Theorem 2, with poles and zeros at the cusps and satisfying a certain natural condition, is an eta quotient, and in particular it is modular. Here we allow $f$ to have a weight $k$. This extends results found in $[\mathrm{K}]$. In Section 4 we explain how GMFs arise in the theory of orbifolds and in particular how our results apply to many vertex operator algebras, including $V^{\natural}$.

\section{§2. GMFs with empty divisor}

In this section we show that Conjecture (2) is true in case the logarithmic derivative of $f$ is a cusp-form of weight 2 , i.e. if $f$ has no poles or zeros in $\mathcal{H} \cup \mathbf{Q} \cup\{\infty\}$. Indeed, we establish two stronger versions of the Conjecture under this assumption, one in which $\Gamma_{0}(N)$ is replaced by an arbitrary subgroup of $S L(2, \mathbf{Z})$ of finite index, and one in which the Fourier coefficients of $f$ are rational numbers and are $p$-integral for almost all primes $p$.

Fix a subgroup $\Gamma \subseteq S L_{2}(\mathbf{Z})$ of finite index, which at the outset is not necessarily a congruence subgroup. Let $M$ be the least positive integer with $\left(\begin{array}{cc}1 & M \\ 0 & 1\end{array}\right) \in \Gamma$. We let $f$ be a GMF on $\Gamma$ of weight zero such that $f$ is holomorphic and non-vanishing at infinity, so that $f$ has a Fourier expansion of the form

$$
f(\tau)=\sum_{n \geq 0} a(n) q^{n / M} \quad(\tau \in \mathcal{H})
$$

with $a(0) \neq 0$. 
TheOREM 1. Assume that $f$ has no poles or zeros in $\mathcal{H} \cup \mathbf{Q} \cup\{\infty\}$. Furthermore, suppose that $a(0)=1$ is normalized and that each $a(n)(n \in$ $\mathbf{N}_{0}$ ) is a rational integer. Then $f=1$ is constant.

Proof. Since $f$ has no zeros or poles, it follows from Theorem 2 in [KM1] that the function

$$
g=\frac{\theta f}{f}
$$

(as usual, $\theta=q^{1 / M} \frac{d}{d q^{1 / M}}=\frac{M}{2 \pi i} \frac{d}{d \tau}$ ) is a holomorphic cusp form of weight 2 on $\Gamma$. (Note that for this, the "parabolic" property of $f$ assumed in [KM1] is not needed.) We write

$$
g=\sum_{n=1}^{\infty} b(n) q^{n / M}
$$

By $[\mathrm{ES}],[\mathrm{BKO}], f$ has an infinite product expansion

$$
f(\tau)=\prod_{n=1}^{\infty}\left(1-q^{n / M}\right)^{c(n)} \quad(|q|<1)
$$

where the $c(n)$ are uniquely determined complex numbers and complex powers as usual are defined in terms of the principal branch of the complex logarithm. Moreover, one has the relations

$$
\begin{gathered}
b(n)=-\sum_{d \mid n} d c(d), \\
n c(n)=-\sum_{d \mid n} \mu(d) b\left(\frac{n}{d}\right) .
\end{gathered}
$$

In particular, since the $a(n)$ are rational, the $c(n)$ are rational. We claim that under our hypothesis, the $c(n)$ in fact are integers (compare also [ES]). Indeed, using the expansion

$$
\left(1-q^{1 / M}\right)^{c}=\sum_{n \geq 0}\left(\begin{array}{l}
c \\
n
\end{array}\right) q^{n / M} \quad(|q|<1, c \in \mathbf{R})
$$

where

$$
\left(\begin{array}{l}
c \\
n
\end{array}\right)=\frac{c(c-1) \cdots(c-n+1)}{n !}
$$


we find from (3) that

$$
\begin{array}{r}
a(n)=(-1)^{\nu_{1}+\cdots+\nu_{n}} \sum_{\nu_{1}+2 \nu_{2}+\cdots+n \nu_{n}=n}\left(\begin{array}{c}
c(1) \\
\nu_{1}
\end{array}\right)\left(\begin{array}{c}
c(2) \\
\nu_{2}
\end{array}\right) \cdots\left(\begin{array}{c}
c(n) \\
\nu_{n}
\end{array}\right) \\
=-c(n)+(-1)^{\nu_{1}+\cdots+\nu_{n-1}} \\
\times \sum_{\nu_{1}+2 \nu_{2}+\cdots+(n-1) \nu_{n-1}=n}\left(\begin{array}{c}
c(1) \\
\nu_{1}
\end{array}\right)\left(\begin{array}{c}
c(2) \\
\nu_{2}
\end{array}\right) \cdots\left(\begin{array}{c}
c(n-1) \\
\nu_{n-1}
\end{array}\right)
\end{array}
$$

where both sums range nonnegative integers $\nu_{i}$. Since the $a(n)$ are integers by hypothesis and $\left(\begin{array}{l}\alpha \\ n\end{array}\right) \in \mathbf{Z}$ for $\alpha \in \mathbf{Z}$, our claim follows by induction.

We now apply the Rankin-Selberg estimate to the Fourier coefficents $b(n)$, which holds even though $\Gamma$ is not necessarily a congruence subgroup [S]. Then

$$
b(n) \ll_{f, \epsilon} n^{4 / 5+\epsilon} \quad(\epsilon>0),
$$

hence we conclude from (5) that

$$
n c(n) \ll_{f, \epsilon} \sum_{d \mid n} d^{4 / 5+\epsilon} \ll n^{4 / 5+\epsilon} \sigma_{0}(n) \ll_{\epsilon} n^{4 / 5+2 \epsilon} \quad(\epsilon>0),
$$

where $\sigma_{0}(n)$ is the number of positive divisors of $n$. Since the $c(n)$ are integers, it follows that $c(n)=0$ for $n \gg 0$.

At this point there are several ways to complete the proof of the Theorem. From (4) we conclude that the $b(n)$ are bounded, hence the RankinSelberg zeta function of $g$, defined for $\sigma=\Re(s)>2$ by

$$
R_{g}(s)=\sum_{n \geq 1} b(n)^{2} n^{-s}
$$

is convergent for $\sigma>1$. On the other hand, it is well-known that $R_{g}(s)$ has a pole at $s=2$ of residue (up to a non-zero multiple) equal to the square $\langle g, g\rangle$ of the Petersson norm of $g$. Hence $g=0$, i.e. $f=1$. Alternatively, the condition $c(n)=0, n \gg 0$, together with (3) shows that $f$ is a rational function of $q^{1 / M}$. It is shown in [KM2, Section 6] that the real axis is a natural boundary for a nonconstant component of any vector-valued modular form. In particular, this applies to $f$ and allows us to again conclude that $f$ is constant. 
TheOREM 2. Assume that $f$ has no poles or zeros in $\mathcal{H} \cup \mathbf{Q} \cup\{\infty\}$. Assume furthermore that $\Gamma$ is a congruence subgroup and that the Fourier coefficents $a(n)\left(n \in \mathbf{N}_{0}\right)$ are rational and are p-integral for all but a finite number of primes $p$. Then $f$ is constant.

Proof. The proof follows the general lines of Theorem 1, but is considerably harder in the present case. We may, and shall, assume that $a(0)=1$ and that $\Gamma$ is the principal congruence subgroup of level $M$. We retain notation from the previous proof, in particular $f$ continues to have the product representation $(3)$.

For a prime $p$, set $\mathbf{Z}_{(p)}:=\mathbf{Z}_{p} \cap \mathbf{Q}$. Let $S$ be the set of primes such that $a(n) \in \mathbf{Z}_{(p)}$ for all $n$ if, and only if, $p \notin S$. Thus $S$ is finite by hypothesis. Since $f$ is normalized, we see that $b(n) \in \mathbf{Z}_{(p)}$ for all $n$ if $p \notin S$. Since $\left(\begin{array}{l}\alpha \\ n\end{array}\right) \in \mathbf{Z}_{(p)}$ for $\alpha \in \mathbf{Z}_{(p)}$, as easily follows from $p$-adic continuity, we also conclude as in the proof of Theorem 1 that $c(n) \in \mathbf{Z}_{(p)}$ for all $n$ if $p \notin S$.

By [Sh, Thm. 3.52], the space $S_{2}(\Gamma)$ of weight 2 cusp-forms on $\Gamma$ has a basis of functions whose Fourier coefficients are integers, hence there exists $A \in \mathbf{N}$ such that

$$
A b(n) \in \mathbf{Z}
$$

for all $n$. Choosing $A$ minimal, it follows that the primes dividing $A$ are contained in $S$.

Let $n \in \mathbf{N}$ and write $n=n_{1} m$ where $n_{1}, m \in \mathbf{N}, n_{1}$ has only prime divisors in $S$ and $m$ is coprime to all primes in $S$. Since $A n c(n) \in \mathbf{Z}$ by (5) and (6) and $c(n)$ is $p$-integral for $p \notin S$, it follows that

$$
A n_{1} c(n) \in \mathbf{Z}
$$

for all $n$. By the Eichler-Shimura-Deligne estimates for Fourier coefficients of weight 2 cusp-forms on a congruence subgroup, we have

$$
b(n) \ll_{f, \epsilon} n^{1 / 2+\epsilon} \quad(\epsilon>0),
$$

hence by (5) we obtain

$$
n c(n) \ll_{f, \epsilon} \sum_{d \mid n} d^{1 / 2+\epsilon} \leq n^{1 / 2+\epsilon} \sigma_{0}(n) \ll_{\epsilon} n^{1 / 2+2 \epsilon} .
$$


If $c(n) \neq 0$, we therefore conclude from (7) and (9) that

$$
\frac{m}{A} \leq \frac{m}{A} \cdot A n_{1}|c(n)|=n|c(n)| \ll_{f, \epsilon}\left(n_{1} m\right)^{1 / 2+\epsilon} \quad(\epsilon>0),
$$

and hence

$$
m \ll_{f, \epsilon} A^{\frac{2}{1-2 \epsilon}} n_{1}^{\frac{1+2 \epsilon}{1-2 \epsilon}} \quad(0<\epsilon<1 / 2) .
$$

Taking e.g. $\epsilon=1 / 4$ (which is sufficient for our purposes), we thus infer that for $n=n_{1} m$ with $n_{1}$ and $m$ as above we either must have

$$
c(n)=0
$$

or else $m$ is bounded by

$$
m \leq C \cdot A^{4} \cdot n_{1}^{3}
$$

where $C$ is an absolute constant depending only on $f$.

If $S=\left\{p_{1}, \ldots, p_{t}\right\}$ and $n_{1}=p_{1}^{\alpha_{1}} \cdots p_{t}^{\alpha_{t}}\left(\alpha_{\nu} \geq 0\right)$, then (11) in particular implies that

$$
m \leq K\left(\alpha_{1}, \ldots, \alpha_{t}\right)
$$

where

$$
K\left(\alpha_{1}, \ldots, \alpha_{t}\right):=C \cdot p_{1}^{4\left(e+\alpha_{1}\right)} \cdots p_{t}^{4\left(e+\alpha_{t}\right)}
$$

and $e$ is an appropriately chosen positive integer.

We will now argue that the Hecke $L$-series

$$
L_{g}(s):=\sum_{n \geq 1} b(n) n^{-s}
$$

attached to $g$, which is known to converge absolutely for $\sigma>3 / 2$, is in fact absolutely convergent for $\sigma>\frac{13}{10}$. From this one easily derives a contradiction (unless $g=0$, i.e. $f=1$ ) by again comparing with the Rankin-Selberg series $R_{g}(s)$.

Indeed, from (10) and (12) it follows for any $\epsilon>0$ that

$$
\begin{aligned}
\sum_{n \geq 1}|c(n)| n^{-\sigma+1} & =\sum_{\alpha_{1}, \ldots, \alpha_{t} \geq 0, m \leq K\left(\alpha_{1}, \ldots, \alpha_{t}\right)}\left|c\left(m p_{1}^{\alpha_{1}} \cdots p_{t}^{\alpha_{t}}\right)\right|\left(m p_{1}^{\alpha_{1}} \cdots p_{t}^{\alpha_{t}}\right)^{-\sigma+1} \\
& \sum_{f, \epsilon}\left(m p_{1}^{\alpha_{1}} \cdots p_{t}^{\alpha_{t}}\right)^{-\sigma+1 / 2+\epsilon} \\
& =\sum_{\alpha_{1}, \ldots, \alpha_{t} \geq 0}\left(p_{1}^{\alpha_{1}} \cdots p_{t}^{\alpha_{t}}\right)^{-\sigma+1 / 2+\epsilon}\left(\sum_{m \leq K\left(\alpha_{1}, \ldots, \alpha_{t}\right)} m^{-\sigma+1 / 2+\epsilon}\right)
\end{aligned}
$$


where in the second line we have used (8).

Suppose that $\frac{1}{2}+\epsilon<\sigma<\frac{3}{2}+\epsilon$. Then for any $K>2$ we clearly have

$$
\sum_{m \leq K} m^{-\sigma+1 / 2+\epsilon} \leq 1+\int_{2}^{K} x^{-\sigma+1 / 2+\epsilon} d x \ll K^{-\sigma+3 / 2+\epsilon}
$$

Therefore for such $\sigma$ we obtain that

$$
\sum_{n \geq 1}|c(n)| n^{-\sigma+1} \ll_{f, \epsilon} \sum_{\alpha_{1}, \ldots, \alpha_{t} \geq 0}\left(p_{1}^{\alpha_{1}} \cdots p_{t}^{\alpha_{t}}\right)^{-\sigma+1 / 2+\epsilon} K\left(\alpha_{1}, \ldots, \alpha_{t}\right)^{-\sigma+3 / 2+\epsilon}
$$

The sum on the right-hand side is equal to

$$
\left(p_{1} \cdots p_{t}\right)^{4 e(-\sigma+3 / 2+\epsilon)} \sum_{\alpha_{1}, \ldots, \alpha_{t} \geq 0}\left(p_{1}^{\alpha_{1}} \cdots p_{t}^{\alpha_{t}}\right)^{-5 \sigma+13 / 2+5 \epsilon}
$$

which is $\ll_{f, \epsilon} 1$ for $\sigma>\frac{13}{10}+\epsilon$.

We therefore deduce that the Dirichlet series

$$
\sum_{n \geq 1} c(n) n^{-s+1}
$$

is absolutely convergent on any line close to the right of the line $\sigma=\frac{13}{10}$, hence must be absolutely convergent for $\sigma>\frac{13}{10}$.

Now observe that (4) is equivalent to

$$
L_{g}(s)=-\zeta(s)\left(\sum_{n \geq 1} c(n) n^{-s+1}\right) \quad(\sigma>3 / 2)
$$

and so $L_{g}(s)$ indeed is absolutely convergent for $\sigma>\frac{13}{10}$. Therefore by (8) we infer that

$$
R_{g}(\sigma) \ll_{f, \epsilon} \sum_{n \geq 1}|b(n)| n^{-\sigma+1 / 2+\epsilon}<\infty
$$

for $\sigma>\frac{9}{5}+\epsilon$ and any $\epsilon>0$. Since $R_{g}(s)$ has a pole at $s=2>\frac{9}{5}$ of residue $\langle g, g\rangle$ as already stated above, it follows that $g=0$, i.e. $f=1$. This completes the proof of Theorem 2. 


\section{§3. GMFs with cuspidal divisor}

In this section we always take the subgroup $\Gamma$ to be $\Gamma_{0}(N)$. We let $f$ be a GMF on $\Gamma_{0}(N)$ of some integral weight $k$, and consider the possibility that the GMF $f$ has all of its poles and zeros at the cusps of $\Gamma$. We will prove Conjecture (2) assuming an additional mild cusp condition.

Recall that a complete set of representatives of the cusps of $\Gamma_{0}(N)$ is given by the numbers $a / c$ where $c$ runs over positive divisors of $N$, and for given $c$, $a$ runs through integers with $1 \leq a \leq N,(a, N)=1$ that are inequivalent modulo $(c, N / c)$.

The width of the cusp $a / c$ is given by

$$
w=w_{a / c}=\frac{N}{\left(c^{2}, N\right)} .
$$

If $f$ is a GMF of integral weight $k$ on $\Gamma_{0}(N)$, then at the cusp $a / c, f$ has a Fourier expansion

$$
(c \tau+d)^{-k} f\left(\frac{a \tau+b}{c \tau+d}\right)=\sum_{n \gg-\infty} a(n) q^{n / w}
$$

convergent in a small punctured neighborhood of $q=0$, where $b, d$ are integers with $a d-b c=1$.

If $f$ is not identically zero, recall that the order $\operatorname{ord}_{a / c} f$ of $f$ at $a / c$ is defined as $n_{0}$ where $n_{0}$ is the smallest integer such that $a\left(n_{0}\right) \neq 0$. We introduce the following hypothesis:

(14) Condition C: for each $c \mid N$, the order $\operatorname{ord}_{a / c} f$ is independent of $a$.

THEOREM 3. Let $f$ be a GMF of integral weight $k$ on $\Gamma_{0}(N)$, and suppose that the poles and zeros of $f$ are supported at the cusps. Suppose further that the Fourier coefficients satisfy the same conditions as in Theorem 2 and that $f$ satisfies condition $C$. Then $f$ is an eta-quotient, i.e. there are integers $M \neq 0$ and $m_{t}(t \mid N)$ such that

$$
f^{M}(\tau)=\prod_{t \mid N} \Delta(t \tau)^{m_{t}}
$$

where $\Delta(\tau)$ is the classical discriminant. In particular, $f$ is modular. 
Proof. We have

$$
\Delta(\tau)=q \prod_{n \geq 1}\left(1-q^{n}\right)^{24} .
$$

For given integers $m_{t}(t \mid N)$ put

$$
F(\tau)=\prod_{t \mid N} \Delta(t \tau)^{m_{t}} .
$$

Then $F$ is on $\Gamma_{0}(N)$ and as is well-known (cf. [M] and references therein), one has

$$
\operatorname{ord}_{a / c} F=w_{a / c} \sum_{t \mid N} \frac{(t, c)^{2}}{t} m_{t}
$$

with $w_{a / c}$ given by (13).

Note that $w_{a / c} \frac{(t, c)^{2}}{t}$ is indeed an integer for $t \mid N, \operatorname{ord}_{a / c} F$ is independent of $a$, and $F$ is normalized with integral Fourier coefficients.

We want to show that the $m_{t}$ can be chosen such that

$$
\operatorname{ord}_{a / c} F=m h_{a / c}
$$

for all cusps $a / c$ of $\Gamma_{0}(N)$. Here, $h_{a / c}$ is the order of $f^{12}$ at $a / c$ and $m$ is an appropriate non-zero integer depending only on $f$. Note that $h_{a / c}$ is an integer by assumption and is independent of $a$. It is sufficient to prove that the rational square matrix

$$
A_{N}=\left((t, c)^{2}\right)_{t|N, t| c}
$$

of size $\sigma_{0}(N)$ and with the divisors of $N$ arranged in some fixed order, is invertible.

If $N=N_{1} N_{2}$ with $\left(N_{1}, N_{2}\right)=1$, then clearly

$$
A_{N}=A_{N_{1}} \otimes A_{N_{2}}
$$

is the Kronecker product of $A_{N_{1}}$ and $A_{N_{2}}$ (the arrangements of the orders of the divisors of $N_{1}$ and $N_{2}$ in $A_{N_{1}}$ and $A_{N_{2}}$ have to be made compatible with those in $A_{N}$ ). Hence

$$
\left\|A_{N}\right\|=\left\|A_{N_{1}}\right\|^{\sigma\left(N_{2}\right)}\left\|A_{N_{2}}\right\|^{\sigma\left(N_{1}\right)}
$$

( $\|A\|$ means the determinant of a square matrix $A)$. 
It is therefore sufficient to show that $A_{p^{\alpha}}$ is invertible for all primes $p$ and all $\alpha \geq 1$. Fix a prime $p$ and put $x:=p^{2}$. Then we find from the definition that

$$
A_{p^{\alpha}}=\left(\begin{array}{ccccc}
1 & 1 & 1 & \cdots & 1 \\
1 & x & x & \cdots & x \\
1 & x & x^{2} & \cdots & x^{2} \\
\vdots & \vdots & & & \\
1 & x & x^{2} & \cdots & x^{\alpha}
\end{array}\right)
$$

Suppose that $\alpha \geq 2$. By Laplace's rule, expanding the determinant with respect to the first column, we find that

$$
\begin{aligned}
\left|A_{p^{\alpha}}\right| & =\left|\begin{array}{cccc}
x & x & \cdots & x \\
x & x^{2} & \cdots & x^{2} \\
\vdots & \vdots & & \vdots \\
x & x^{2} & \cdots & x^{\alpha}
\end{array}\right|-\left|\begin{array}{cccc}
1 & 1 & \cdots & 1 \\
x & x^{2} & \cdots & x^{2} \\
\vdots & \vdots & & \vdots \\
x & x^{2} & \cdots & x^{\alpha}
\end{array}\right|+\left|\begin{array}{cccc}
1 & 1 & \cdots & 1 \\
x & x & \cdots & x \\
\vdots & \vdots & & \vdots \\
x & x^{2} & \cdots & x^{\alpha}
\end{array}\right| \mp \cdots \\
& =\left(x^{\alpha}-x^{\alpha-1}\right)\left|\begin{array}{ccccc}
1 & 1 & 1 & \cdots & 1 \\
1 & x & x & \cdots & x \\
1 & x & x^{2} & \cdots & x^{2} \\
\vdots & \vdots & \vdots & & \vdots \\
1 & x & x^{2} & \cdots & x^{\alpha-1}
\end{array}\right| \mp
\end{aligned}
$$

since the third and higher terms of the sum on the right-hand side of (16) clearly vanish.

We thus obtain

$$
\left|A_{p^{\alpha}}\right|=p^{2 \alpha-2}\left(p^{2}-1\right)\left|A_{p^{\alpha-1}}\right| \quad(\forall \alpha \geq 2) .
$$

Since

$$
\left|A_{p}\right|=p^{2}-1
$$

we therefore see by induction that $A_{p^{\alpha}}$ is invertible for all $\alpha \geq 1$. We thus have established formula (15), with an appropriate $m$.

Let $k_{1}$ be the weight of $F$. Since $\Delta$ does not vanish on $\mathcal{H}$, we find from the valence formula applied to $F$ that the sum of the orders of $F$ at the different cusps of $\Gamma_{0}(N)$ is equal to

$$
\frac{k_{1}}{12}\left[\Gamma(1): \Gamma_{0}(N)\right]
$$


On the other hand, by [KM1, Lemma 3.5] the valence formula is also valid for the GMF $f^{12}$ of weight $12 k$. Since $f$ has no zeros on $\mathcal{H}$ by assumption, we therefore deduce from (15) that

$$
k_{1}=12 m k \text {. }
$$

Letting $M=12 m$ we see that $f^{M} / F$ is a GMF satisfying all of the assumptions of Theorem 2. We conclude from the Theorem that $f^{M}=F$, as required. This completes the proof of Theorem 3 .

\section{§4. Vertex operator algebras and GMFs}

The purpose of this section is to review some results from the theory of orbifolds, i.e. the action of finite groups on vertex operator algebras, and explain how GMFs arise in this context. We refer to [DLM] and [DM1] for further background and details. However, a detailed understanding of VOA theory will not be required in order to understand the results of this section. We work in the following setting ${ }^{2}$ :

$V$ is a $C_{2}$-cofinite, holomorphic vertex operator algebra over $\mathbf{C}$ with decomposition

$$
V=\mathbf{C 1} \oplus V_{1} \oplus V_{2} \cdots
$$

into $L(0)$-eigenspaces and central charge $c$ divisible by 24 .

$G \subseteq$ Aut $V$ is a finite group of automorphisms of $V$.

For example, the Moonshine Module $V^{\natural}$ satisfies these conditions (loc. cit.) with $c=24$ and $G$ the Monster simple group $M$. Each homogeneous space $V_{n}$ is a finite-dimensional $G$-module (with $V_{0}=\mathbf{C 1}$ the trivial module), and we denote by $a_{n}$ the character of $G$ afforded by $V_{n}$. Then for $h \in G$ we set

$$
Z(h, \tau)=q^{-c / 24} \sum_{n=0}^{\infty} a_{n}(h) q^{n}
$$

Note that if $h=1$ then (18) is just the graded dimension of $V$ :

$$
Z(1, \tau)=q^{-c / 24} \sum_{n=0}^{\infty}\left(\operatorname{dim} V_{n}\right) q^{n}=q^{-c / 24}+\cdots
$$

\footnotetext{
${ }^{2}$ See the footnote on p. 121 regarding the adjective 'holomorphic' that occurs in (17).
} 
Zhu proved [Z] that (19) is a modular function (weight zero) on the full modular group $S L(2, \mathbf{Z})$, possibly with a character (which necessarily has order dividing 3). It is expected that all of the trace functions (18) are modular functions on a congruence subgroup of $S L(2, \mathbf{Z})$, but this remains open.

Next we record some general properties of the trace functions (18) which follow from elementary facts about complex representations of a finite group. Because $V$ is holomorphic it admits a unique normalized, non-degenerate, invariant bilinear form $\langle\rangle,([\mathrm{L}])$, moreover the form is both symmetric [FHL] and $G$-invariant. This implies that each $a_{n}$ is an orthogonal representation of $G$, and hence

$$
\text { the coefficients } a_{n}(h) \text { are real algebraic integers in }
$$

$$
\mathbf{Z}\left[\zeta_{N}\right] \text {, where } \zeta_{N}=e^{2 \pi i / N} \text { and } N \text { is the order of } h \text {. }
$$

For an integer $d$ coprime to $N$, let $\sigma_{d}$ be the Galois automorpism of $\mathbf{Q}\left(\zeta_{N}\right)$ induced by $\zeta_{N} \mapsto \zeta_{N}^{d}$. Denote by $Z(h, \tau)^{\sigma_{d}}$ the $q$-expansion obtained by replacing each coefficient $a_{n}(h)$ by $a_{n}(h)^{\sigma_{d}}$. Then $a_{n}(h)^{\sigma_{d}}$ is the character of $G$ given by the $d$ th Adams operation $\psi^{d}\left(a_{n}\right)$, and

$$
Z(h, \tau)^{\sigma_{d}}=q^{-c / 24} \sum_{n=0}^{\infty} \psi^{d}\left(a_{n}\right)(h) q^{n}=Z\left(h^{d}, \tau\right) .
$$

In particular, the following are equivalent:

(a) $Z(h, \tau)=Z\left(h^{d}, \tau\right)$ whenever $(N, d)=1$

(b) each $a_{n}$ restricts to a rational representation of $\langle h\rangle$

(c) $Z(h, \tau)$ has integral coefficients.

The next result is deeper, and is proved in [DLM]:

each $Z(h, \tau)$ is holomorphic in $\tau$, i.e. it is a holomorphic function in the complex upper half-plane $\mathcal{H}$.

Now we need the twisted sectors. For each element $g \in G$, a $g$-twisted sector is a certain vector space analogous to (17) which admits an irreducible action of $V$ by $g$-twisted operators. We will not need the details of this definition, only the consequences established in [DLM]. Namely, for each $g$ there is a unique $g$-twisted sector (up to isomorphism), denoted by $V(g)$. 
If $g=1$ then $V(1)$ is nothing but the vertex operator algebra $V$. The $L(0)$ operator induces a grading on $V(g)$ of the form

$$
V(g)=\sum_{n=0}^{\infty} V(g)_{n / N+\lambda_{g}}
$$

where $\lambda_{g}$ (the conformal weight of $V(g)$ ) lies in $\mathbf{Q}$ and $V(g)_{\lambda_{g}} \neq 0$. There is a projective action of the centralizer $C_{G}(g)=\{h \in G \mid g h=h g\}$ on $V(g)$. That is, $V(g)$ determines a 2-cocycle $\alpha_{g} \in Z^{2}\left(C_{G}(g), \mathbf{C}^{*}\right)$ such that each $V(g)_{n / N+\lambda_{g}}$ affords a representation of the twisted group algebra $\mathbf{C}^{\alpha_{g}}\left[C_{G}(g)\right]$. We denote the corresponding character by $a_{n, g}$. For each pair of commuting elements $g, h \in G$, regarded as elements of $\mathbf{C}^{\alpha_{g}}\left[C_{G}(g)\right]$, we define

$$
Z(g, h, \tau)=q^{-c / 24+\lambda_{g}} \sum_{n=0}^{\infty} a_{n, g}(h) q^{n / N} .
$$

A little care is needed with this definition. Replacing $\alpha_{g}$ by a cohomologous 2-cocycle will give a different graded trace which will differ from (24) by an overall nonzero scalar. Because $V(1)$ is identifed with $V, Z(1, h, \tau)$ is just different notation for $Z(h, \tau)$. All of these $q$-expansions enjoy property (22), i.e. they are holomorphic in $\mathcal{H}$. Note that taking $h=1$ in (24) yields the graded dimension of $V(g)$. Thus,

$$
Z(g, 1, \tau)=q^{-c / 24+\lambda_{g}} \sum_{n=0}^{\infty} \operatorname{dim} V(g)_{n / N+\lambda_{g}} q^{n / N}
$$

has nonnegative integral coefficients.

Within the setting in which we are working, the main modular-invariance result of [DLM] says the following ${ }^{3}$ :

Let $\gamma=\left(\begin{array}{ll}a & b \\ c & d\end{array}\right) \in S L(2, \mathbf{Z})$. There is a nonzero scalar $\epsilon(\gamma, g, h)$

independent of $\tau$ such that

$$
Z(g, h, \gamma \tau)=\epsilon(\gamma, g, h) Z((g, h) \gamma, \tau)
$$

where $(g, h) \gamma=\left(g^{a} h^{c}, g^{b} h^{d}\right)$.

\footnotetext{
${ }^{3}$ The reader should have no difficulty distinguishing between the lower left entry of matrices such as $\gamma$, and the central charge, both of which we denote by $c$.
} 
From now on we take $G=\langle h\rangle$ to be the cyclic group generated by an element $h$ of order $N$. Choosing $\gamma \in \Gamma_{1}(N)$ and $g=1$ in (27), and setting $\chi_{h}(\gamma)=\epsilon(\gamma, 1, h)$, we obtain

$$
Z(h, \gamma \tau)=\chi_{h}(\gamma) Z(h, \tau) .
$$

So (27) and (28) together show that

$$
Z(h, \tau) \text { is a GMF of weight zero on } \Gamma_{1}(N) \text { with character } \chi_{h} \text {. }
$$

If instead we take $\gamma \in \Gamma_{0}(N)$ in (27), we find that (28) still holds as long as $Z(h, \tau)$ is replaced on the right hand side by $Z\left(h^{d}, \tau\right)$. From $(21)$ we can conclude:

Suppose that $Z(h, \tau)$ has integral coefficients. Then

$$
Z(h, \gamma \tau)=\chi_{h}(\gamma) Z(h, \tau), \gamma \in \Gamma_{0}(N),
$$

and $Z(h, \tau)$ is a GMF of weight zero on $\Gamma_{0}(N)$ with character $\chi_{h}$.

We now show that if $Z(h, \tau)$ is a GMF on $\Gamma_{0}(N)$ (regardless of the nature of the Fourier coefficients), then $Z(h, \tau)$ satisfies Condition $\mathrm{C}(14)$. A cusp $a / c$ of $\Gamma_{0}(N)$ with $c \mid N$ corresponds to a double coset

$$
\Gamma_{0}(N)\left(\begin{array}{ll}
a & b \\
c & d
\end{array}\right)\left\langle \pm\left(\begin{array}{ll}
1 & 1 \\
0 & 1
\end{array}\right)\right\rangle
$$

and we may choose $a, d$ to be coprime to $N$. If we make such a choice, and set $\gamma=\left(\begin{array}{ll}a & b \\ c & d\end{array}\right)$, then $Z(h, \gamma \tau)$ is a $q$-expansion for $Z(h, \tau)$ at the cusp $a / c$. Hence (27) tells us that

$$
Z(h, \gamma \tau) \sim Z\left(h^{c}, h^{d}\right) \sim Z\left(h^{c}, h\right)^{\sigma_{d}},
$$

where we have used $\sim$ to indicate that the relevant $q$-expansions differ only by an overall nonzero scalar. In particular, the order of $Z(h, \tau)$ at the cusp $a / c$ is independent of $a$, as required.

We collect some of our results together in the next Theorem.

THEOREM 4. Let $V$ be a holomorphic vertex operator algebra (17), and suppose that $h$ is an automorphism of $V$ of finite order $N$. Then the following hold:

(a) $Z(h, \tau)$ is a $G M F$ on $\Gamma_{1}(N)$;

(b) If $Z(h, \tau)$ has integral coefficients then it is a GMF on $\Gamma_{0}(N)$;

(c) If $Z(h, \tau)$ is a $G M F$ on $\Gamma_{0}(N)$ then it satisfies Condition $C$. 
Corollary 5. Let the assumptions be as in Theorem 4. If $Z(h, \tau)$ has integral coefficients and its zeros are at the cusps of $\Gamma_{0}(N)$ then $Z(h, \tau)$ is an eta-quotient. In particular, $Z(h, \tau)$ is a modular function of weight zero on $\Gamma_{0}(N)$.

The Corollary follows from Theorems 3 and 4 together with (22) and applies, for example, to lattice vertex operator algebras $V_{L}([\mathrm{FLM}])$ where $L$ is an even, self-dual lattice and $h$ arises from a fixed-point-free automorphism of $L$.

Concerning the particular case of the Moonshine Module $V^{\natural}$, we observe that condition $(21)$ (b) holds in this case. Indeed, perusal of the character table of the Monster group [A] shows that an irreducible complex character of $M$ is either rational or not self-dual. Therefore, all real representations of $M$ are rational, and our assertion follows from (20). (A slightly different, but equivalent, discussion is given in [DM2, p. 118].) As a result, each $Z(h, \tau)$ has integral Fourier coefficents by $(21)$, hence by Theorem 4 it is a GMF on some $\Gamma_{0}(N)$ which satisfies Condition C. Hence,

Corollary 6. Suppose that $V$ is the Moonshine Module $V^{\natural}$ and $h \in$ $M$ has order $N$. Then $Z(h, \tau)$ is a GMF on $\Gamma_{0}(N)$ which has rational integral Fourier coefficients and which satisfies Condition $C$.

In this case, a number of elements $h \in M$ satisfy the condition that for some constant $\alpha, Z(h, \tau)+\alpha$ has its pole(s) and zero(s) at the cusps (cf. $[\mathrm{CN}]$ ). Removal of the requirement of a cuspidal divisor in Theorem 3 would, by Corollary 6 , prove the modularity of all Monster trace functions. Of course, in this case the resulting modular function is not necessarily an eta-quotient.

\section{REFERENCES}

[A] J. Conway et al., Atlas of Finite Groups, Clarendon Press, Oxford, 1985.

[B] R. Borcherds, Monstrous moonshine and monstrous Lie superalgebras, Invent. Math., 109 (1992), 405-444.

[BKO] J. Bruinier, W. Kohnen and K. Ono, The arithmetic of the values of modular functions and the divisors of modular forms, Compos. Math., 140 (2004), no. 3, $552-566$.

[CN] J. Conway and S. Norton, Monstrous Moonshine, Bull. Lond. Math. Soc., 12 (1979), 308-339. 
[DLM] C. Dong, H. Li, and G. Mason, Modular-Invariance of Trace Functions in Orbifold Theory and Generalized Moonshine, Comm. Math. Phys., 214 (2000), 1-56.

[DM1] C. Dong and G. Mason, Vertex operator algebras and Moonshine: A survey, Adv. Stud. in Pure Math., 24 (1996), 101-136.

[DM2] C. Dong and G. Mason, Monstrous moonshine of higher weight, Acta Math., 185 (2000), 101-121.

[ES] W. Eholzer and N.-P. Skoruppa, Product expansions of conformal characters, Phys. Lett., B 388 (1996), 82-89.

[FHL] I. Frenkel, Y.-Z. Huang, and J. Lepowsky, On axiomatic approaches to vertex operator algebras and modules, Mem. Amer. Math. Soc. 104, 1993.

[FLM] I. Frenkel, J. Lepowsky, and A. Meurman, Vertex Operator Algebras and the Monster, Academic Press, San Diego, 1988.

[K] W. Kohnen, On a certain class of modular functions, Proc. Amer. Math. Soc., 133 (2005), no. 1, 65-70.

[KM1] M. Knopp and G. Mason, Generalized modular forms, J. Number Theory, 99 (2003), 1-18.

[KM2] M. Knopp and G. Mason, Vector-Valued Modular Forms and Poincaré Series, Ill. J. Math., 48 (2004), no. 4, 1345-1366.

[L] H. Li, Symmetric invariant bilinear forms on vertex operator algebras, J. Pure and Appl. Alg., 96 (1994), 279-297.

[M] Y. Martin, Multiplicative $\eta$-quotients, Trans. Amer. Math. Soc., 348 (1996), no. $12,4825-4856$.

[S] A. Selberg, On the Estimation of Fourier Coefficients of Modular Forms, Proc. Symp. Pure Math. Vol. VIII, Amer. Math. Soc., Providence R.I., 1965.

[Sh] G. Shimura, Introduction to the Arithmetic Theory of Automorphic Functions, Publ. Math. Soc. Jap. 11, Iwanami Shoten, 1971.

[Z] Y. Zhu, Modular-invariance of characters of vertex operator algebras, J. Amer. Math. Soc., 9 (1996), 237-302.

Winfried Kohnen

Mathematisches Institut der Universität

INF 288, D-69120 Heidelberg

Germany

winfried@mathi. uni-heidelberg.de

Geoffrey Mason

Department of Mathematics

University of California at Santa Cruz

California 95064

USA

gem@cats.ucsc.edu 